\title{
Local Chain Dynamics of syndiotactic Poly(methyl methacrylate) Studied by Fluorescence Depolarization Method
}

\author{
Junichi Horinaka, Keiko Ono, and Masahide Yamamoto* \\ Division of Polymer Chemistry, Graduate School of Engineering, Kyoto University, \\ Sakyo-ku, Kyoto 606, Japan
}

(Received November 16, 1994)

\begin{abstract}
Poly(methyl methacrylate)s ( $s$-PMMAs) with anthracene in the middle of a main chain were synthesized, and their local motions in dilute solutions were examined by the fluorescence depolarization method. The local motion of two kinds of $s$-PMMAs with different molecular weights was examined in several low viscosity solvents. The mean relaxation time was measured and the activation energy was evaluated for the local motion of each $s$-PMMA. First, the effect of the solvent quality on the local chain dynamics was observed. The value of the relaxation time and its activation energy became smaller as the solvent became better one. Secondly the molecular weight dependence of the local motion in poor solvents differs from that in good solvents. In poor solvents, the relaxation time of the local chain became longer as the molecular weight increased, while in a good solvent, the local motion was not influenced by the change of molecular weight. These observations could be explained by the concept of the segment density.

KEY WORDS Fluorescence Depolarization / syndiotactic Poly(methyl methacrylate) / Polymer Chain Dynamics / Segment Density / Molecular Weight Effect / Excluded Volume Effect /
\end{abstract}

Polymer chains in dilute solutions undergo micro-Brownian motion and have the relaxation times of nano to subnano seconds. ${ }^{1,2}$ The local chain dynamics has been extensively studied. Several models to express the local motion have been proposed, ${ }^{4-9}$ e.g., a model proposed by Hall and Helfand. ${ }^{4}$ As the experimental techniques to examine the local motion, neutron scattering, ${ }^{10}$ NMR, ${ }^{11,29}$ $\mathrm{ESR},{ }^{12,30}$ dielectric relaxation, ${ }^{13,14}$ and fluorescence depolarization ${ }^{16-19,28}$ etc. have been utilized.

We have examined the local motion of polymer chains by the fluorescence depolarization method. ${ }^{19-26}$ The advantage of this method is that the orientational autocorrelation function can be directly observed. We have measured the local chain dynamics of the chain center for such polymers as poly(methyl methacrylate) (PMMA), ${ }^{19-22,26}$ poly( $\alpha$-methylstyrene), ${ }^{23,25}$ and polystyrene, ${ }^{23,25}$ etc. For
PMMA, at high viscosity or at low temperatures, the discrete conformational jump models $^{5,6}$ were in good agreement with the experimental data. ${ }^{20}$ Static $^{27}$ or dynamic chain stiffness parameter of PMMA was estimated and compared with those of other poly(alkyl methacrylate)s. $^{22}$ The dependence of local motion on the stereoregularity of a polymer chain was also examined ${ }^{26}$ for syndiotactic- and isotactic-PMMA.

Monnerie et al. studied the molecular weight dependence of the relaxation time of local motion for polystyrene solutions by NMR. ${ }^{29}$ They calculated the isotropic correlation times of polystyrene in $10 \mathrm{wt} \%$ solution which had a time range of $10^{-10}-10^{-9} \mathrm{~s}$. They concluded that overall rotatory diffusion is less effective on the relaxation times of local motion in high molecular weight polystyrenes, and that the correlation times remain constant in $M_{w}>10000$. In the present study, we examined 
the local chain motion of syndiotactic-poly(methyl methacrylate) ( $s$-PMMA) in lowviscosity solvents by the fluorescence depolarization method. The relaxation time was measured for two kinds of $s$-PMMAs with an $M_{w}$ of $2.0 \times 10^{4}$ and $1.5 \times 10^{5}$ in $0.1 \mathrm{wt} \%$ solutions. We evaluated the activation energy for the local motion, and discussed the effects of the solvent quality and the molecular weight.

\section{EXPERIMENTAL}

Anthracene-labeled $s$-PMMAs were prepared by the living anionic polymerization initiated by (1,1-diphenyl hexyl)lithium and the living ends were coupled by 9,10-bis(bromomethyl)anthracene (Figure 1). The detailed synthetic technique was described elsewhere. ${ }^{20,31}$ Table I shows the molecular weights determined by GPC and racemo fractions of the obtained $s$-PMMAs by ${ }^{13} \mathrm{C}$ NMR. The solvents used for the measurements were acetonitrile (Nacalai Tesque), butyl chloride (Nacalai Tesque), acetone (Nacalai Tesque), toluene (Wako Pure Chemical Industries), benzene (Wako Pure Chemical Industries), and chloroform (Nacalai Tesque). The intrinsic viscosity in each solvent was measured by capillary viscometry. The PMMA sample (Polyscience) for the viscometry had an $M_{w}$ of $4.7 \times 10^{4}, M_{w} / M_{n}$ of 1.95 and a racemo fraction of dyad of 0.81 . We evaluated the chain expansion factor, $\bar{\alpha}_{\eta}^{3}$, by the dilution method, where $\bar{\alpha}_{\eta}^{3}$ is the ratio of intrinsic viscosity of a solution to that of the solution at the $\theta$ condition. In this study, the $\theta$ condition was used in butyl chloride at $35.0^{\circ} \mathrm{C}^{32}$ The timeresolved fluorescence anisotropy ratio was measured by the single-photon counting system $^{24}$; a time-to-amplitude converter (TAC Ortec Model 457), a discriminator (Ortec Model 436), a constant fraction discriminator (Ortec Model 583), and a multichannel analyzer (Norland Ino-Tech 5300). A diode laser PLP-01 (Hamamatsu Photonics, wavelength: $411 \mathrm{~nm}$, FWHM: $c a .50 \mathrm{ps}$ ) was used as a light



Figure 1. Anthracene-labeled s-PMMA sample.

Table I. Molecular weights, its distributions, and racemo fractions of labeled $s$-PMMAs

\begin{tabular}{ccccc}
\hline Sample & $M_{n} \times 10^{-4}$ & $M_{w} \times 10^{-4}$ & $M_{w} / M_{n}$ & $f_{\mathrm{r}}$ \\
\hline$s$-PMMA1 & 2.1 & 2.3 & 1.11 & 0.93 \\
$s$-PMMA2 & 12.8 & 15.0 & 1.18 & 0.93 \\
\hline
\end{tabular}

source and a microchannel plate-photomultiplier tube as a detector. The repetition rate of the diode laser was $1 \mathrm{MHz}$ and the singlephoton counting system was used in the mode that the start and stop in TAC were reversed. The excitation light was polarized vertically, and the parallel and perpendicular fluorescence components to the plane of polarization were measured. We used the cutoff filters V-42 and Y-44 (HOYA) for observation. The FWHM of the total instrumental function was $c a .300 \mathrm{ps}$. The effect of solvent quality on the chain dynamics was examined for $s$ PMMA2 in all six solvents, and the molecular weight effect between poor and good solvents was compared for $s$-PMMA1 and 2 in acetonitrile, butyl chloride, benzene, and chloroform. The polymer concentration of each sample solution was prepared to be $c a \cdot 10^{-5} \mathrm{M}$. The parallel component, $I_{\mathrm{vv}}(t)$, and the perpendicular component, $I_{\mathrm{VH}}(t)$, were measured alternatively to avoid data distortions due to the time drift. The anisotropy ratio, $r(t)$, is defined as eq 1 .

$$
r(t)=\left(I_{\mathrm{Vv}}(t)-G I_{\mathrm{VH}}(t)\right) /\left(I_{\mathrm{Vv}}(t)+2 G I_{\mathrm{VH}}(t)\right)
$$

where $G$ is the compensating factor. We fitted the equation in which eq 2 was convoluted with the instrumental function to the experimental 
anisotropy data by nonlinear-least-squares method. ${ }^{20}$ It is known empirically that eq 2 represents the experimental data quite well. ${ }^{20}$

$$
r(t)=r_{0}\left[x \exp \left(-t / T_{1}\right)+(1-x) \exp \left(-t / T_{2}\right)\right]
$$

The mean relaxation time $T_{\mathrm{m}}$ is defined by eq 3 , and the value $T_{\mathrm{m}}$ can be calculated by eq 4 with the parameters $T_{1}, T_{2}$, and $x$ obtained from the fitting.

$$
\begin{aligned}
T_{\mathrm{m}} & =r_{0}^{-1} \int_{0}^{\infty} r(t) \mathrm{d} t \\
& =x T_{1}+(1-x) T_{2}
\end{aligned}
$$

\section{RESULTS AND DISCUSSION}

\section{Solvent Effect}

Table II shows the chain expansion factors of $s$-PMMA chain, $\bar{\alpha}_{\eta}^{3}$, solvent viscosity, $\eta$, and its activation energy, $E_{\eta}$, for each solvent. ${ }^{33}$ The solvent viscosity of each solvent is lower than $1 \mathrm{cP}$ at $35^{\circ} \mathrm{C}$ and these low viscosity systems can be treated with the theory of Kramers' diffusion limit. ${ }^{1,34}$ The chain expansion factors indicate that the order of solvent quality from good to poor one for $s$-PMMA is chloroform, benzene, toluene, acetone, butyl chloride, and acetonitrile.

We evaluated the activation energy of the local motion by the theory of Kramers' diffusion limit. When a particle with a frictional

Table II. Solvent viscosities at $35^{\circ} \mathrm{C}$, activation energies, and chain expansion factors of $s$-PMMA at $35^{\circ} \mathrm{C}$

\begin{tabular}{lcccc}
\hline \multirow{2}{*}{ Solvent } & \multicolumn{1}{c}{$\eta$} & $E_{\eta}$ & \\
\cline { 2 - 3 } & $\mathrm{cP}$ & $\mathrm{kcal} \mathrm{mol}^{-1}$ & & \\
\hline Chloroform & 0.941 & 1.81 & 2.36 \\
Benzene & 0.526 & 2.50 & 1.99 \\
Toluene & 0.492 & 2.11 & 1.73 \\
Acetone & 0.283 & 1.65 & 1.41 \\
Butyl chloride & 0.387 & 1.69 & 1.00 \\
Acetonitrile & 0.311 & 1.66 & 0.93 \\
\hline
\end{tabular}

coefficient, $\zeta$, passes over an energy barrier with the height $E^{*}$, the velocity coefficient, $k$, is represented by eq 5 .

$$
k \propto \zeta^{-1} \exp \left(-E^{*} / R T\right)
$$

where $R$ is the gas constant and $T$ is absolute temperature. Since $T_{\mathrm{m}}$ is proportional to the reciprocal of $k$ and the Stokes' law, $\zeta \propto \eta$, holds, $T_{\mathrm{m}}$ can be represented by

$$
T_{\mathrm{m}} / \eta=A \exp \left(E^{*} / R T\right)
$$

Figure 2 shows the Arrhenius plots of $T_{\mathrm{m}} / \eta$ for $s$-PMMA2 solutions. A microscopic aggregation $^{25}$ occurred in the $\theta$ solvent (butyl chloride) in the temperature range lower than the $\theta$ temperature by ten degrees or more. Figure 2 indicates that the relaxation time is influenced by the solvent quality. Figure 3 shows the reduced relaxation time, $T_{\mathrm{m}} / \eta$, at the $\theta$ temperature, $35^{\circ} \mathrm{C}$, against the chain expansion factor, $\bar{\alpha}_{\eta}^{3}$. $T_{\mathrm{m}} / \eta$ becomes smaller with the chain expansion, i.e., the local chain relaxation time of $s$-PMMA in a poor solvent is slower than that in a good solvent. The slopes of the lines in Figure 2 correspond to the activation energy of local motion, $E^{*}$. Figure 4 shows the relationship between the activation energy and the chain expansion factor. The fact that $E^{*}$ becomes smaller with the chain expansion is

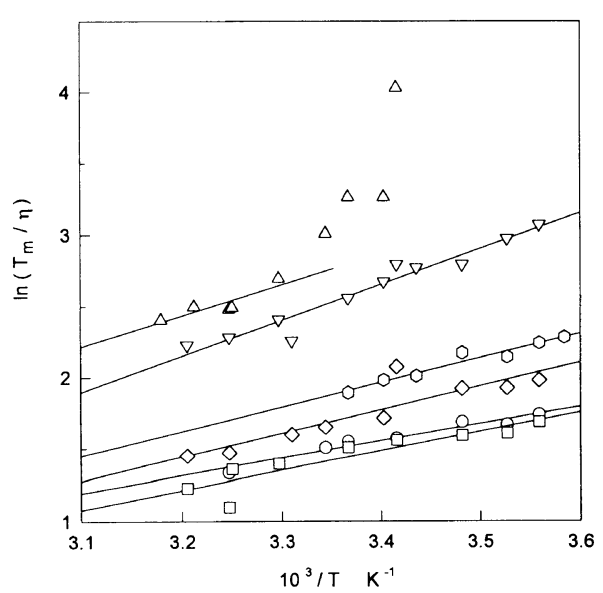

Figure 2. $\ln \left(T_{\mathrm{m}} / \eta\right) v s .1 / T$ plots for $s$-PMMA2 solutions: $\bigcirc$, chloroform; $\square$, benzene; $\diamond$, toluene; $\square$, acetone; $\triangle$, butyl chloride; $\nabla$, acetonitrile. 


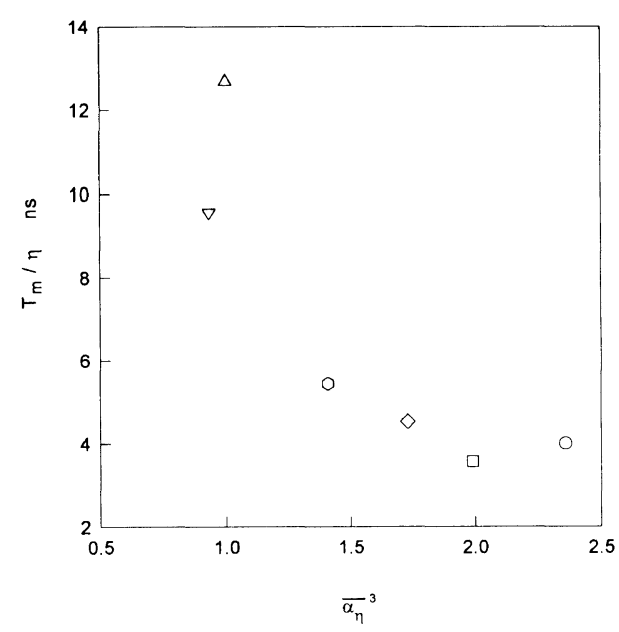

Figure 3. The relationship between relaxation times and chain expansion factors for $s$-PMMA2 solutions at $35^{\circ} \mathrm{C}$. Symbols are the same as in Figure 2.

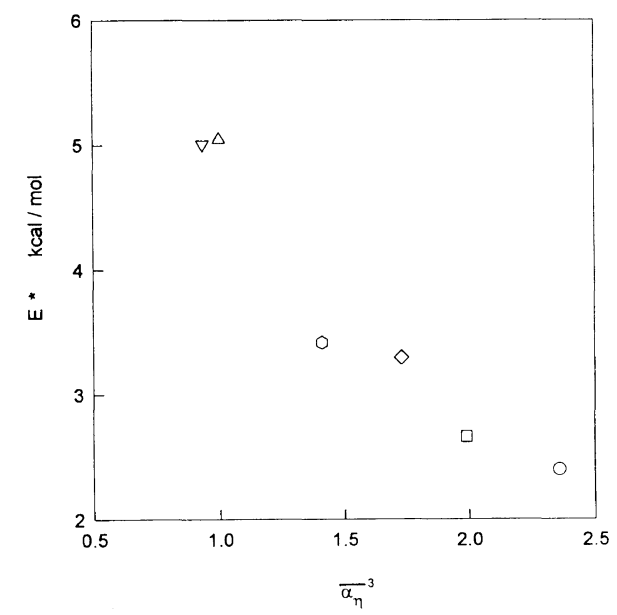

Figure 4. The relationship between activation energies and chain expansion factors for $s$-PMMA2 solutions. Symbols are the same as in Figure 2.

similar to the case of the relaxation time. The observed chain expansion dependence of the relaxation time and of the activation energy is explained by the degree of segment density about the chain center. The $s$-PMMA chain shrinks in a poor solvent and the segment density is rather high. The neighboring segments around the probe in a poor solvent suppress the local segmental motion, and this hindrance results in larger values of relaxation time and activation energy than those in good solvents. That is, the high segment density about the chain center leads to the suppression of the chain mobility. The relationship between the segment density and the chain mobility was also examined for polystyrenes; polystyrene, $\operatorname{poly}(\alpha$-methylstyrene $)$, and poly ( $p$-methylstyrene). ${ }^{25}$ For all three samples, the relaxation time and the activation energy decrease with the chain expansion in the same way as for $s$-PMMA.

\section{Molecular Weight Effect}

Figure $5 \mathrm{a}-\mathrm{d}$ shows the plotting of $T_{\mathrm{m}} / \eta$ against $1 / T$ for $s$-PMMA1 and $s$-PMMA2 in such a solvent, acetonitrile (a), butyl chloride (b), benzene (c), and chloroform (d). We found that in poor solvents ( $a$ and $b$ ), the reduced relaxation time depends strongly on the molecular weight. At a fixed temperature, $T_{\mathrm{m}} / \eta$ for $s$-PMMA2 was larger than that for $s$-PMMA1. On the other hand, in good solvents (c and $d$ ), the relaxation time was almost independent of the molecular weight, and the relaxation times between $s$-PMMA1 and 2 were almost the same within the experimental error. Table III shows the activation energy of $s$-PMMA1 and 2 in four solvents. Similarly to the relaxation time, the activation energy increases with the molecular weight in a poor solvent, while in a good solvent it is independent of the molecular weight. In poor solvents such as acetonitrile or butyl chloride, the value of $E^{*}$ for $s$-PMMAl is $c a .3$ $\mathrm{kcal} \mathrm{mol}^{-1}$, and that for $s$-PMMA2 is $c a$. $5 \mathrm{kcal} \mathrm{mol}^{-1}$. On the other side, the value of $E^{*}$ in a good solvent such as benzene or chloroform is almost constant at ca. $2.5 \mathrm{kcal}$ $\mathrm{mol}^{-1}$. The difference in the molecular weight effect on the chain dynamics either in poor solvents or in good solvents can be also explained by taking account of the segment density. In the vicinity of $\theta$ point, polymer segments behave as if they do not interact with each other, because the excluded volume effect 

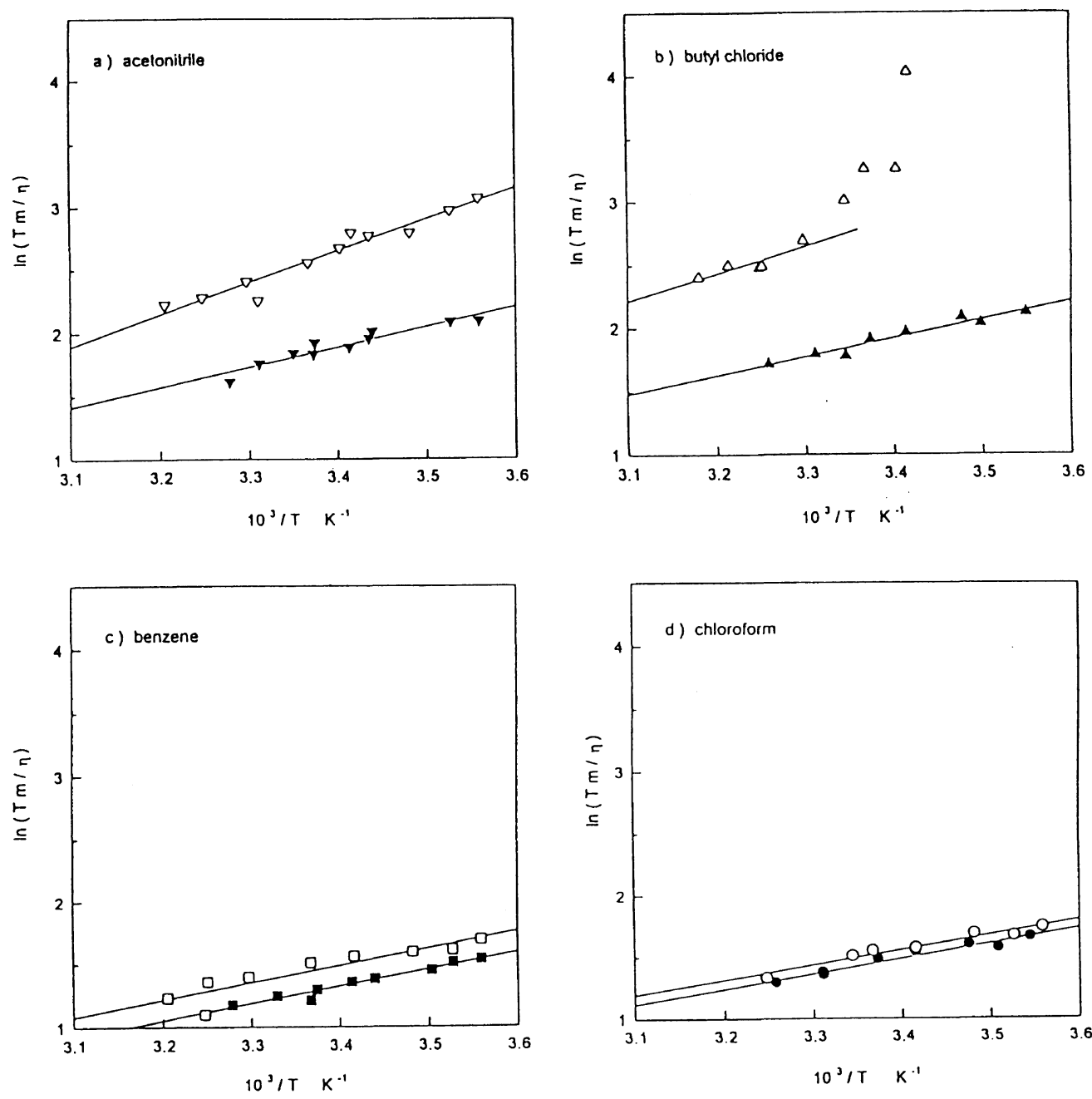

Figure 5. Molecular weight effect on the chain mobility for $s$-PMMA1 (black) and $s$-PMMA2 (white): a) acetonitrile, b) butyl chloride, c) benzene, and d) chloroform.

Table III. Activation energy, $E^{*}\left(\mathrm{kcal} \mathrm{mol}^{-1}\right)$, for $s$-PMMA1 and 2 solutions

\begin{tabular}{lcc}
\hline \multicolumn{1}{c}{ Solvent } & $s$-PMMA1 & $s$-PMMA2 \\
\hline Acetonitrile & 3.2 & 5.0 \\
Butyl chloride & 2.9 & 5.0 \\
Benzene & 2.6 & 2.7 \\
Chloroform & 2.4 & 2.4
\end{tabular}

mostly vanishes. It is valid to suppose that the polymer chain, nearly in the $\theta$ condition, takes a Gaussian distribution and the segment density about the chain center increases with the molecular weight. ${ }^{17,35}$ The local motion essentially consists of internal rotational conformation transitions. It is considered that under the condition of high segment density in a poor solvent, intramolecular segments hinder the chain rotation, and the local chain dynamics becomes slow. In good solvents, $s$-PMMA chain expands sufficiently and the segment density is kept nearly constant because of the excluded volume effect when the molecular weight is changed. ${ }^{17}$ Hence, the local 
chain dynamics in good solvents is almost independent of the molecular weight.

Waldow et al. ${ }^{17}$ performed random walk calculations for their polyisoprene chain, and in their calculations the local segment density about the chain-center segment was shown. They applied a self-avoiding random walk simulation for the chain in good solvents and also a regular random walk for the chain in poor $(\theta)$ solvents. Their findings indicate that in the self-avoiding walk (good solvent model) the segment density is kept almost constant irrespective of molecular weight and that in the regular random walk ( $\theta$ solvent model) the density increases with the molecular weight. Moreover, the self-avoiding walk shows a less congested environment than the regular random walk. Although they did not expect their calculation to be quantitatively accurate, their calculations strongly support our explanation based on the segment density.

\section{CONCLUSION}

The local chain dynamics of two $s$-PMMAs with different molecular weights was examined by the fluorescence depolarization method. The dynamics was affected by the solvent quality, i.e., the relaxation time became shorter with improvement of the solvent quality. In poor solvents; the dynamics became slower in the PMMA with a higher molecular weight, while in good solvents, the local chain mobility was not influenced by the molecular weight. These observations can be explained by the degree of the segment density about the chain center. In poor solvents, the segment density increases with the increase of molecular weight keeping a Gaussian distribution, while in good solvents the segment density is kept constant due to the excluded volume effect.

Acknowledgments. We are indebted to Dr. Takashi Sasaki, Fukui University, for the preparation of the anthracene-labeled $s$ PMMAs. This work was supported by a
Grant-in-Aid for Scientific Research on Priority Areas, Photochemical Reactions (No. 06239107) from the Ministry of Education, Science, and Culture of Japan.

\section{REFERENCES}

1. E. Helfand, J. Chem. Phys., 54, 4651 (1971).

2. H. Yamakawa, Macromolecules, 10, 692 (1977).

3. T. A. Weber and E. Helfand, J. Phys. Chem., 87, 2881 (1977).

4. C. K. Hall and E. Helfand, J. Chem. Phys., 77, 3275 (1982).

5. A. A. Jones and W. H. Stockmayer, J. Polym. Sci., Polym. Phys. Ed., 15, 847 (1977).

6. J. L. Viovy, L. Monnerie, and J. C. Brochon, Macromolecules, 16, 1845 (1983).

7. J. T. Bendler and R. Yaris, Macromolecules, 11, 650 (1978).

8. B. Valeur, J. Jarry, F. Geny, and L. Monnerie, J. Polym. Sci., Polym. Phys. Ed., 13, 667 (1975); B. Valeur, L. Monnerie, and J. Jarry, ibid., 13, 675 (1975).

9. J. Skolnick and R. Yaris, Macromolecules, 15, 1041 (1982).

10. J. S. Higgins and A. Maconnachie, "Methods of Experimental Physics," Vol. 23, Part C, K. Sköld and D. L. Price, Ed., Academic Press, London, 1987, p 287.

11. S. Glowinkowski, D. J. Gisser, and M. D. Ediger, Macromolecules, 23, 3520 (1990).

12. R. T. Bailey, A. M. North, and R. A. Pethrick, "Molecular Motion in High Polymers," Clarendon Press, Oxford, 1981.

13. a) S. Mashimo, Macromolecules, 9, 91 (1976); b) S. Mashimo, P. Winsor, R. H. Cole, K. Matsuo, and W. H. Stockmayer, Macromolecules, 19, 682 (1986).

14. K. Adachi, Macromolecules, 23, 1816 (1990).

15. B. Valeur and L. Monnerie, J. Polym. Sci., Polym. Phys. Ed., 14, 11 (1976).

16. M. D. Ediger, Ann. Rev. Phys. Chem., 42, 225 (1991).

17. D. A. Waldow, B. S. Johnson, P. D. Hyde, M. D. Ediger, T. Kitano, and K. Ito, Macromolecules, 22, 1345 (1989).

18. D. A. Waldow, M. D. Ediger, Y. Yamaguchi, Y. Matsushita, and I. Noda, Macromolecules, 24, 3147 (1991).

19. T. Sasaki and M. Yamamoto, Macromolecules, 22, 4009 (1989).

20. T. Sasaki, M. Yamamoto, and Y. Nishijima, Macromolecules, 21, 610 (1988).

21. T. Sasaki, K. Nawa, M. Yamamoto, and Y. Nishijima, Eur. Polym. J., 25, 79 (1989).

22. T. Sasaki, H. Arisawa, and M. Yamamoto, Polym. J., 23, 103 (1991). 
23. S. Yokotsuka, Y. Okada, Y. Tojo, T. Sasaki, and M. Yamamoto, Polym. J., 23, 95 (1991).

24. K. Ono, Y. Okada, S. Yokotsuka, S. Ito, and M. Yamamoto, Polym. J., 26, 199 (1994).

25. K. Ono, Y. Okada, S. Yokotsuka, T. Sasaki, and M. Yamamoto, Macromolecules, 27, 6482 (1994).

26. K. Ono, T. Sasaki, M. Yamamoto, Y. Yamasaki, $\mathrm{K}$. Ute, and $\mathrm{K}$. Hatada, Macromolecules, to be submitted.

27. M. Fujii, K. Nagasaka, J. Shimada, and H. Yamakawa, Macromolecules, 16, 1613 (1983).

28. P. D. Hyde, D. A. Waldow, M. D. Ediger, T. Kitano, and K. Ito, Macromolecules, 19, 2533 (1986).

29. F. Lauprêtre, C. Noël, and L. Monnerie, J. Polym.
Sci., Polym. Phys. Ed., 15, 2127 (1977).

30. A. T. Bullock, G. G. Cameron, and P. M. Smith, J. Phys. Chem., 77, 1635 (1973).

31. T. Sasaki, M. Yamamoto, and Y. Nishijima, Makromol. Chem., Rapid Commun., 7, 345 (1986).

32. R. G. Kirste and G. V. Schulz, Z. Phys. Chem., 27, 301 (1961).

33. J. A. Riddick and W. B. Bunger, "Techniques of Chemistry, Vol. 2: Organic Solvents," 3rd ed, WileyInterscience, New York, N. Y., 1970.

34. H. A. Kramers, Physica, 7, 284 (1940).

35. D. A. Waldow, B. S. Johnson, C. L. Babiarz, M. D. Ediger, T. Kitano, and K. Ito, Polym. Commun., 29, 296 (1988). 